\title{
Vulnerabilidade ambiental urbana nas regiões metropolitanas do Ceará
}

\author{
Christiane Luci Bezerra Alves \\ Anderson da Silva Rodrigues \\ Nayara de Sousa Rodrigues
}

\begin{abstract}
Resumo
Um dos importantes movimentos de conformação do sistema de cidades no Brasil tem sido a fragmentação institucional da gestão metropolitana, que transfere aos estados a possibilidade de criação de suas regiões metropolitanas. Ao mesmo tempo, estudos sobre vulnerabilidade ambiental têm se destacado em contextos nos quais a análise dos sistemas econômicos e sociais é cada vez mais repensada a partir de suas conexões ambientais. Este ensaio se propõe a identificar a vulnerabilidade ambiental urbana em regiões metropolitanas do estado do Ceará pela construção de um índice sintético de vulnerabilidade (IVA). A análise do IVA aponta níveis de vulnerabilidade semelhantes entre as regiões metropolitanas, todavia, com dispersões distintas. Enquanto a RMF e RM Cariri apresentam maior homogeneidade, a RMS apresenta menor percentual de municípios abaixo da média regional e maior dispersão dos valores, o que leva ao questionamento sobre a existência de efeitos de espraiamento desta região metropolitana.
\end{abstract}

Palavras-chave | Ceará; índice de vulnerabilidade; regiões metropolitanas; vulnerabilidade ambiental urbana.

Classificação JEL | H70 Q56 R58

\section{Urban environmental vulnerability in the metropolitan regions of Ceará}

\begin{abstract}
One of the important movements in shaping the system of cities in Brazil has been the institutional fragmentation of metropolitan management, which transfers to the states the possibility of creating their metropolitan regions. At the same time, studies on environmental vulnerability have been highlighted in the context where the analysis of economic and social systems is increasingly rethought based on their environmental connections. This essay proposes to identify urban environmental vulnerability in metropolitan regions of the state of Ceará, by creating a synthetic vulnerability index (IVA). The VAT analysis shows similar levels of vulnerability among metropolitan regions, however, with different dispersions. While for the
\end{abstract}


RMF and RM Cariri are more homogeneous, the RMS has a lower percentage of municipalities below the regional average and a greater dispersion of values, which leads to questions about the existence of spreading effects in this metropolitan region.

Keywords | Ceará; metropolitan regions; urban environmental vulnerability; vulnerability index.

JEL Classification | H70 Q56 R58

\section{Vulnerabilidad ambiental urbana en las regiones metropolitanas de Ceará}

\section{Resumen}

Uno de los movimientos importantes en la configuración del sistema de ciudades en Brasil ha sido la fragmentación institucional de la gestión metropolitana, que transfiere a los estados la posibilidad de crear sus regiones metropolitanas. Al mismo tiempo, los estudios sobre vulnerabilidad ambiental se han destacado en contextos en los que el análisis de los sistemas económicos y sociales se repiensa cada vez más en función de sus conexiones ambientales. Este ensayo propone identificar la vulnerabilidad ambiental urbana en las regiones metropolitanas del estado de Ceará mediante la construcción de un índice de vulnerabilidad sintética (IVA). El análisis del IVA apunta niveles similares de vulnerabilidad entre las regiones metropolitanas, sin embargo, con diferentes dispersiones. Mientras que el RMF y el RM Cariri presentan mayor homogeneidad, el RMS tiene un menor porcentaje de municipios por debajo del promedio regional y una mayor dispersión de valores, lo que genera dudas sobre la existencia de efectos de propagación de esta región metropolitana.

Palabras clave | Ceará; índice de vulnerabilidad; regiones metropolitanas; vulnerabilidad ambiental urbana.

Clasificación JEL | H70 Q56 R58

\section{Introdução}

A expansão da rede urbana nas últimas décadas do século XX desenha alguns claros movimentos da conformação do sistema de cidades no Brasil. O primeiro deles é determinado pelo rápido e intenso mecanismo de urbanização que acompanha as últimas etapas do processo de substituição de importações brasileiro e da constituição da matriz industrial nacional, especialmente na dinâmica de forte crescimento dos anos 1970, onde grandes centros urbanos ganham contorno de metrópoles. O segundo encontra-se associado à fragmentação institucional da gestão metropolitana, facilitada pelos novos arranjos federativos proporcionados pela Constituição Federal de 1988, que transfere aos estados a possibilidade de 
criação de suas regiões metropolitanas (BRASIL, 1988). É nesse contexto que se firmam e consolidam um conjunto de novas metrópoles regionais.

Cabe destacar que o processo de integração econômica que acompanha os processos de metropolização não é acompanhado por alterações/ampliações significativas na oferta de serviços públicos nem pela descentralização destes na mesma escala em que se intensificam os fluxos de pessoas, bens e serviços, característicos dessas escalas de integração, apesar de certa distribuição e hierarquização de funcionalidades intrametropolitanas, determinando fortes pressões sobre os centros urbanos regionais, especialmente aquelas que contribuem para algum tipo de vulnerabilidade ambiental. Nesse sentido, tem-se constatado "com o crescimento acelerado das grandes cidades e com os processos de conurbação que nelas frequentemente ocorrem, certos problemas urbanos são potencializados, adquirindo um caráter de vulnerabilidade ambiental, propenso a processos antropogênicos induzidos" (BARCELLOS; OLIVEIRA, 2008, p.2).

Os estudos sobre a vulnerabilidade ambiental têm se destacado no contexto onde a análise dos sistemas econômicos e sociais é cada vez mais repensada a partir de suas conexões ambientais, como parte de um novo entendimento sistêmico e multidimensional do desenvolvimento, no qual as diferentes ciências têm contribuído para a interpretação de problemáticas que captem aspectos da melhoria da qualidade de vida e bem-estar das populações, em prol da sustentabilidade e do desenvolvimento territorial.

Deste modo, o presente ensaio se propõe ao entendimento do padrão de vulnerabilidade ambiental urbana presente em regiões metropolitanas do estado do Ceará, a partir da medição desse fenômeno por meio de um índice sintético de vulnerabilidade, constituído pelas dimensões: habitacional, infraestrutura urbana e pressão sobre o ambiente natural. São tomadas para análise as regiões metropolitanas do estado do Ceará: Região Metropolitana de Fortaleza (RMF); Região Metropolitana do Cariri (RM Cariri) e Região Metropolitana de Sobral (RMS). Como hipótese, espera-se que os núcleos dinâmicos das regiões metropolitanas apresentem menores níveis de vulnerabilidades.

O trabalho encontra-se estruturado em quatro seções, além desta introdução. A segunda seção apresenta os aspectos teórico-conceituais da vulnerabilidade ambiental urbana; na seção três, são abordados os procedimentos metodológicos, com a caracterização das áreas de estudo, dimensões e indicadores selecionados e os procedimentos para a construção do índice sintético de vulnerabilidade; os resultados e discussões são apresentados na seção quatro. Por fim, a quinta seção traz as considerações finais deste ensaio. 


\section{Vulnerabilidade ambiental urbana: aspectos teórico-conceituais}

Apesar da multiplicidade de interpretações as matrizes teóricas da vulnerabilidade ambiental apontam para um primeiro bloco de contribuições, onde a vulnerabilidade está intrinsecamente associada à características físicas do ambiente e as aproximações na literatura inicialmente reconhecem três vetores que caracterizam o fenômeno: exposição ao risco; (in)capacidade de reação; dificuldade de adaptação mediante riscos. Assim, a vulnerabilidade encontra-se constantemente associada ao grau de susceptibilidade de um sistema a fatores intrínsecos ou extrínsecos que sobre ele exercem pressão. Nos fatores intrínsecos incluem-se as características bióticas do meio. Os fatores extrínsecos relacionam-se à exposição do sistema a pressões ambientais atuais e futuras. Dizem respeito, principalmente, ao grau de eficácia de um grupo social em adaptar sua organização às mudanças no ambiente social que incorporam riscos. Envolvem fatores como a possibilidade de um evento catastrófico ocorrer, presença de riscos e danos potenciais, além de perdas objetivas de bens materiais ou vidas (GARCÍA-TORNEL, 1997; VEYRET, 2007).

De certa forma, nessas contribuições, os residentes em ambientes físicos precários e expostos a maiores riscos são mais vulneráveis. Ao mesmo tempo, a capacidade de resposta de indivíduos ou grupos às mudanças no ambiente natural influencia a vulnerabilidade de tais indivíduos ou grupo social (TOMINAGA; SANTORO; AMARAL, 2009). Essa perspectiva se encontra nas análises de organismos multilaterais, como o Escritório das Nações Unidas para a Redução do Risco de Desastres (UNDRR), onde se constata a associação da vulnerabilidade à ideia de resiliência, entendida como a capacidade de um sistema, sociedade ou comunidade de resistir e adaptar-se, para obter um nível aceitável de estrutura e funcionamento (UNDRR, 2004, 2011). Tais orientações evoluem com o debate envolvendo o conceito nos meios políticos e institucionais, em nível mundial, onde ocorre a multiplicação de estudos sobre mudanças climáticas e o efeito estufa e nas interpretações de análise de riscos, que ganham corpo a partir da criação, em 1988, do Painel Intergovernamental sobre Mudança do Clima (Intergovernmental Panel on Climate Change (IPCC, 2001)) $)^{1}$.

De modo geral, por conseguinte, a vulnerabilidade estaria associada à ocorrência de eventos potencialmente adversos e à incapacidade para dar respostas, podendo ser resultado da incapacidade de enfrentar riscos ou da inabilidade de adaptar-se ativamente à situação (CEPAL, 2002; VIGNOLLI, 2006).

1 Resultado de conferência conjunta realizada pela Organização Meteorológica Mundial (Word Meteorological Organization (WMO) e pelo Programa das Nações Unidas para o Meio Ambiente (United Nations Environmental Program (UNEP). 
Em outra linha de contribuições, a capacidade de resposta a situações de mudanças ambientais é determinada por fatores mais sistêmicos, variando de acordo com as possibilidades e condições ambientais, sociais, econômicas, culturais e políticas das populações (ADGER, 2006). A capacidade de adaptação das populações aos diferentes níveis de estresse se encontra estreitamente vinculada a fatores como "riqueza, tecnologia, educação, informação, habilidades, infraestrutura, acesso a recursos e capacidade de gestão" (CONFALONIERI, 2001, p.34).

A trajetória de vulnerabilidades associa-se intimamente, portanto, à (in)capacidade de reação às dificuldades enfrentadas em diferentes dimensões, sob múltiplos determinantes como sociais, econômicos e culturais, a qual é influenciada, adicionalmente, pela presença efetiva e institucional do Estado na promoção de condições adequadas que interfiram na qualidade de vida e interação das populações com o meio natural. Assim,

A vulnerabilidade socioambiental apresenta níveis bastante elevados, por conta da limitada capacidade de adaptação da população frente a eventos extremos adversos, agravada pela vulnerabilidade institucional no sentido de desenvolver planos de gestão dos riscos dirigidos a estes grupos (GAMBA, 2010, p.8).

Portanto, pessoas vulneráveis teriam menos condições de aproveitar as oportunidades oferecidas pelo mercado, o Estado e a sociedade. Tal capacidade de aproveitamento de oportunidades, a que se referem Kaztman et al. (1999) por "posse ou controle de ativos", vincula estreitamente a vulnerabilidade à dimensão social das populações. Ou seja, os autores concentram sua análise na "relação ativos/vulnerabilidade/estrutura de oportunidades", a partir do entendimento de que a disponibilidade de ativos que possibilita o enfrentamento de determinada situação de vulnerabilidade pode ou não ser suficiente, a depender da estrutura de oportunidades que prevalece em determinada área ou região. Ou seja, a capacidade de resposta das famílias depende do tipo de ativos existente e como este é mobilizado para se aproveitarem as oportunidades oferecidas pelo mercado, pelo Estado e pela sociedade em geral (KAZTMAN; FILGUEIRA, 2006).

Em Hogan e Marandola (2006, p. 27), “a vulnerabilidade é associada às desvantagens sociais que produzem e, ao mesmo tempo, são reflexos e produtos da pobreza". O caráter social da vulnerabilidade e sua vinculação com os riscos ambientais também é enfatizado por Deschamps (2004, p. 140), que chama atenção para a estreita relação "entre a localização espacial dos grupos que apresentam desvantagens sociais e aquelas áreas onde há risco de ocorrer algum evento adverso, ou seja, populações socialmente vulneráveis se localizam em áreas ambientalmente vulneráveis". 
Barcellos e Oliveira (2008, p. 10) também enfatizam que o risco ambiental "não se distribui de forma aleatória entre os diversos grupos sociais, mas obedece aos padrões de desigualdade e segregação social que marcam a estruturação das cidades". Além do fenômeno natural, portanto, as estruturas históricas que determinam os processos de ocupações urbanas, mediados por mecanismos de segregação e exclusão de populações a ambientes mais inóspitos e vulneráveis ao risco, ditam a linha social que separa vulneráveis e não vulneráveis dentro da dinâmica urbana.

É nessa orientação que, como campo de pesquisa, para Mendonça (2004, p. 141), "as condições de vida da população passaram a desempenhar importante papel na compreensão dos problemas ambientais urbanos e revelou, ao mesmo tempo, diferenciações claras entre a cidade formal e a cidade informal".

\section{Procedimentos metodológicos}

\section{Delimitação da área de estudo}

A Região Metropolitana de Fortaleza (RMF) foi criada no contexto tecnocrático e centralizador do regime militar, através da Lei Complementar Federal no 14/1973 (BRASIL, 1973) (junto com outras seis metrópoles regionais), sendo composta atualmente pelos municípios: Aquiraz, Cascavel, Caucaia, Chorozinho, Eusébio, Fortaleza, Guaiúba, Horizonte, Itaitinga, Maracanaú, Maranguape, Pacajus, Pacatuba, Paracuru, Paraipaba, Pindoretama, São Gonçalo do Amarante, São Luís do Curu e Trairi (Figura 1). 
Figura 1 - Regiões Metropolitanas do Ceará, 2019

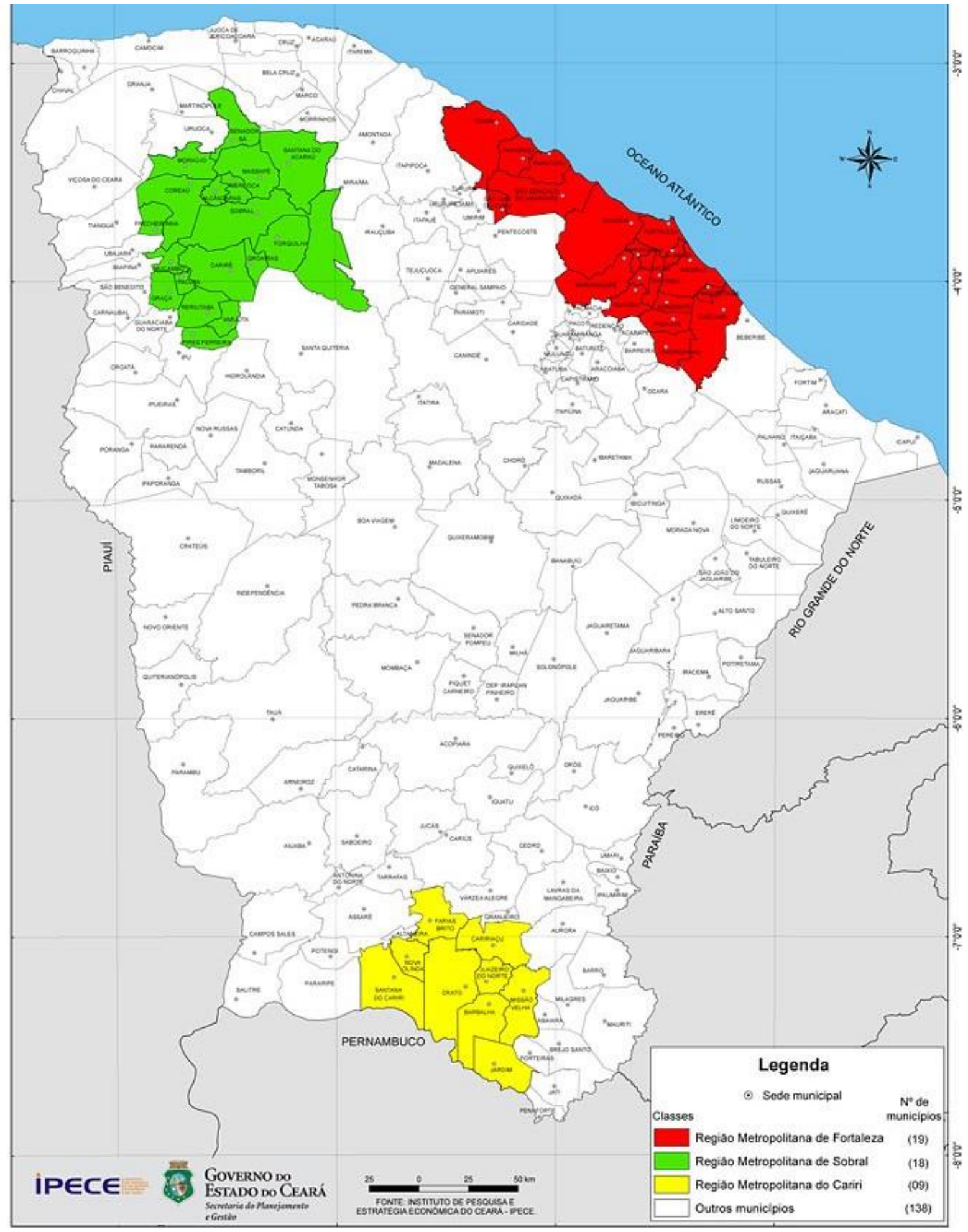

Fonte: Fonte: IPECE (2019).

A RM do Cariri foi instituída através da Lei Complementar Estadual no 78/2009 (CEARÁ, 2009), e a Região Metropolitana de Sobral (RMS), criada por meio da Lei 
Complementar Estadual no 168/2016 (CEARÁ, 2016). Compõem a RM Cariri os municípios de: Barbalha, Caririaçu, Crato, Farias Brito, Jardim, Juazeiro do Norte, Missão Velha, Nova Olinda e Santana do Cariri (Figura 1). É importante ressaltar que a RM Cariri não se caracteriza pela presença de um centro dinâmico formado por apenas um município, mas pela conurbação Crajubar (Crato, Juazeiro do Norte e Barbalha) cujos municípios apresentam características mais homogêneas em relação aos demais, como elevada taxa de urbanização, concentração populacional, de infraestrutura e serviços públicos na região metropolitana (RODRIGUES, ALVES, PINHEIRO, 2017).

Facilitada pela centralidade e dinamização econômica e política recentes da cidade de Sobral, município localizado na mesorregião Noroeste Cearense, com ampla influência em uma grande região de entorno, foi instituída, por meio da Lei Complementar Estadual n ${ }^{\circ}$ 168/2016, a Região Metropolitana de Sobral (RMS) (CEARÁ, 2016). É formada pelos seguintes municípios: Alcântaras, Cariré, Coreaú, Forquilha, Frecheirinha, Graça, Groaíras, Massapê, Meruoca, Moraújo, Mucambo, Pacujá, Pires Ferreira, Reriutaba, Santana do Acaraú, Senador Sá, Sobral e Varjota (Figura 1).

\section{Seleção de indicadores e tratamento e fonte dos dados}

Apesar de reconhecer a multidimensionalidade da vulnerabilidade optou-se, aqui, pela abordagem em três dimensões: habitacional, infraestrutura urbana e pressão sobre o ambiente natural. A inclusão da dimensão habitacional objetiva inferir sobre condições adequadas de moradia, nos indicadores: condição de ocupação (ICO), domicílios em condições sanitárias inadequadas (IDCSI), densidade de moradores por dormitório (IDD) e padrão de revestimento do domicílio (IPRD), que refletem aspectos sociais da vulnerabilidade (ARAÚJO; CÂNDIDO, 2014; BRAGA et al., 2003; MARTINS; CÂNDIDO, 2015; ROLNIK, 2015; IPEA, 2015; IBGE, 2015). $\mathrm{Na}$ infraestrutura urbana, objetiva-se investigar a exclusão da população em relação a serviços e bens públicos considerados essenciais à vida urbana (indicador de cobertura urbana de esgoto - ICUE; indicador de abastecimento de água - IAA; indicador de coleta de lixo domiciliar - ICL). Consideram-se, também, variáveis para medir a vulnerabilidade a partir das condições do entorno e deficiências de infraestrutura que importam em aumento de riscos ambientais, tais como: indicador de condições do entorno - ICE (arborização, lixo acumulado, existência de boca de lobo ou bueiro, esgoto a céu aberto) e indicador de manejo de águas pluviais IMAP. Neste último indicador, as variáveis incorporam risco de enchentes e desmoronamento e estrutura para o enfrentamento destes problemas (ALMEIDA, 2010; GOMES; GOMES, 2017; PEREIRA; VIEIRA, 2016; IPEA, 2015; IBGE, 2015).

A dimensão pressão sobre o ambiente natural (IVPAN) objetiva captar o risco decorrente de pressão das atividades antrópicas sobre o ambiente natural, sobre 
processos naturais de reciclagem de recursos e nutrientes, bem como os riscos inerentes à poluição, queimadas, exposição ao risco de contaminação por substâncias químicas ou agentes biológicos, perda de bem-estar pela redução ou ausência de ambientes conservados etc. (IPEA, 2015; IBGE, 2015; GAMBA, RIBEIRO, 2012; ALMEIDA, 2010; MARTINS, CÂNDIDO, 2015). Nesta dimensão são incluídos os indicadores: emissão de monóxido de carbono veicular (IMCV), resíduos industriais (IRI), focos de queimada por mil hectare (IFQ), número casos de doenças de notificação compulsória (por mil habitantes) causada por agente biológico (IDNC) e indicador de área preservada (IAP).

As fontes de dados são secundárias, extraídas do Anuário Estatístico do Ceará (IPECE, 2011), Censo Agropecuário (IBGE, 2007), Censo Demográfico (IBGE, 2011), Pesquisa Nacional de Saneamento Básico (IBGE, 2010), Inventário de Resíduos Industriais (CEARÁ, 2004) ${ }^{2}$ e inventário nacional de emissões atmosféricas por veículos automotores rodoviários (BRASIL, 2011).

A construção de um índice sintético de vulnerabilidade ambiental urbana requer uma padronização das variáveis de forma que diferenças de escala nas variáveis não provoquem distorções na análise; as variáveis são padronizadas numa escala de zero a um. Tal padronização leva em consideração a relação entre os valores dos municípios do estado do Ceará, onde o menor valor da variável corresponderia a zero na escala (menor vulnerabilidade) e o maior seria o valor 1 (maior vulnerabilidade). Há que se destacar que os valores são relativos, construídos a partir $\mathrm{da}$ realidade estadual, com seus respectivos dados municipais, permitindo a comparação entre municípios e regiões do Ceará, porém, são pouco adequados para comparação com outras regiões ou estados ${ }^{3}$.

\section{A construção do Índice de Sintético de Vulnerabilidade Ambiental (ISVA)}

A construção do Índice de Sintético de Vulnerabilidade Ambiental (ISVA) dos municípios da RM Cariri foi realizada a partir da elaboração de três índices que representam as dimensões relacionadas à vulnerabilidade especificadas anteriormente. Em termos analíticos, o cálculo do ISVA se deu a partir da seguinte equação:

$$
I S V A=\frac{1}{K} \sum_{p=1}^{k} I_{p}
$$

\footnotetext{
2 Os valores foram adaptados com base no crescimento industrial dos respectivos municípios.

3 Isto ocorre em virtude dos valores máximos e mínimos serem fixados com base nos dados de 184 municípios do Estado; para comparações com outras regiões dever-se-iam utilizar dados de todos os municípios do território nacional.
} 
Onde:

ISVA = Índice Sintético de Vulnerabilidade Ambiental

$\mathrm{I}_{\mathrm{p}}=$ valor do p-ésimo índice

$\mathrm{p}=1, \ldots, \mathrm{k}$ (índice)

Cada índice, por sua vez, é formado a partir de um conjunto de indicadores, conforme especificado no Quadro 1. Apesar de possuírem número diverso de variáveis, optou-se por estabelecer pesos iguais entre os indicadores na composição do respectivo índice. Assim, o valor do p-ésimo índice foi calculado, conforme a seguir:

$$
\begin{gathered}
I_{p}=\frac{1}{S} \sum_{q=1}^{s} C_{q} \\
C_{q}=\frac{1}{M} \sum_{j=1}^{m}\left[\frac{1}{N}\left(\sum_{i=1}^{n} \frac{E_{i j}}{\operatorname{Emax}_{i}}\right)\right]
\end{gathered}
$$

Onde:

$\mathrm{Cq}=$ representa a contribuição do q-ésimo indicador no p-ésimo índice dos municípios

$\mathrm{E}_{\mathrm{ij}}=$ escore da i-ésima variável do q-ésimo indicador obtida pelo j-ésimo município;

$\operatorname{Emax}_{i}=$ escore máximo da i-ésima variável do q-ésimo indicador

$\mathrm{i}=1, \ldots, \mathrm{n}$ (variáveis que compõem o indicador " $\mathrm{q}$ ")

$\mathrm{j}=1, \ldots, \mathrm{m}$ (municípios)

$\mathrm{q}=1, \ldots, \mathrm{s}$ (número de indicadores que compõem o p-ésimo índice)

No âmbito do presente trabalho, o índice de vulnerabilidade apresenta escala de zero a 1 , onde o valor zero representa a menor vulnerabilidade relativa entre os municípios estaduais e o valor 1, a maior vulnerabilidade. Para facilitar a análise e exposição dos dados, utilizar-se-á a seguinte escala de vulnerabilidade: baixa vulnerabilidade, para valores do índice entre zero e 0,200 (inclusive); vulnerabilidade intermediária, entre 0,201 e 0,499 (inclusive) e alta vulnerabilidade, no intervalo compreendido entre os 0,500 e 1 (inclusive). 


\section{Resultados e discussões}

\section{Índices de vulnerabilidade habitacional}

A análise do Índice de Vulnerabilidade Habitacional (IVH) utilizou, para a estimação da vulnerabilidade de cada região metropolitana, a média de indicadores ponderada pelo número de domicílios da respectiva região. Em relação à RMF, o valor do índice de Fortaleza teve o maior peso para todos os indicadores, visto ter este município 66\% dos domicílios da região metropolitana. Assim, para o índice parcial de condição de ocupação, que identifica o percentual de domicílios em condição de "cedido ou outra" (visto que aqueles em maior vulnerabilidade habitacional não dispõem de recursos financeiros para alugar ou comprar um imóvel), o nível de vulnerabilidade da RMF é extremamente baixo $(0,04)$, influenciado pelo peso da capital do estado que ficou com valor zero, por apresentar a menor vulnerabilidade relativa em nível estadual. Logo, apenas dois dos 19 municípios da RMF se apresentaram menos vulneráveis que a média da região metropolitana (Fortaleza e Maracanaú). Outro destaque é que tal vulnerabilidade tende a ser menor nos municípios próximos da capital (Caucaia - 0,098; Maracanaú - 0,036; Pacatuba - 0,09; Horizonte - 0,047). Para os municípios mais afastados, a vulnerabilidade tende a aumentar em virtude da mitigação dos efeitos de espraiamento de Fortaleza (Tabela 1). Vale ressaltar que as menores vulnerabilidades coincidem com regiões que apresentam maiores níveis de renda, implicando em melhores condições sociais da população e maior propensão de gastos com moradia.

Em relação ao índice de condições inadequadas de saneamento, apenas Fortaleza, Pacatuba e Maracanaú apresentaram vulnerabilidade menor que a obtida pela RMF, que apresenta vulnerabilidade intermediária. No que tange à classificação, Pacatuba representa o único município com índice na escala de baixa vulnerabilidade $(0,191)$; seis municípios apresentam alta vulnerabilidade (Trairi, São Luiz do Curu, Pindoretama, Chorozinho e Pacajus), estando os demais na faixa de vulnerabilidade intermediária.

O índice de densidade de dormitório atribui a vulnerabilidade aos domicílios com mais de duas pessoas por dormitório. Este índice apresentou a maior vulnerabilidade para a dimensão vulnerabilidade habitacional, classificada como de elevada vulnerabilidade. De forma desagregada, seis dos 19 municípios se classificam na faixa intermediária, apresentando-se os demais altamente vulneráveis. Importante destacar que dentre os municípios com média vulnerabilidade, Fortaleza apresentou o pior desempenho no indicador, possivelmente relacionado aos fatores de atração populacional que pressionam o déficit habitacional, sendo esta uma característica comum entre os núcleos dinâmicos das regiões metropolitanas do país. 
Tabela 1 - Índice de Vulnerabilidade Habitacional e indicadores por região metropolitana do Ceará, 2019

\begin{tabular}{lccccc}
\hline Municípios & ICO & IDCSI & IDD & ITRD & IVH \\
\hline Média RMF & 0,040 & 0,259 & 0,511 & 0,353 & 0,291 \\
Fortaleza & 0,000 & 0,203 & 0,449 & 0,272 & 0,231 \\
Média RMS & 0,237 & 0,326 & 0,644 & 0,235 & 0,360 \\
Sobral & 0,125 & 0,160 & 0,678 & 0,261 & 0,306 \\
Média RM Cariri & 0,139 & 0,378 & 0,643 & 0,175 & 0,334 \\
Barbalha & 0,129 & 0,456 & 0,584 & 0,103 & 0,318 \\
Crato & 0,117 & 0,336 & 0,536 & 0,251 & 0,310 \\
Juazeiro do Norte & 0,082 & 0,330 & 0,693 & 0,148 & 0,313 \\
\hline
\end{tabular}

Fonte: Elaborada pelos autores.

O índice de revestimento objetiva associar a condição social das famílias por meio do padrão de revestimento dos domicílios, sendo considerados vulneráveis os domicílios com outros revestimentos exclusive alvenaria (taipa, tijolo sem revestimento, papelão etc.). A RMF apresenta nível intermediário de vulnerabilidade; apenas São Gonçalo do Amarante é pouco vulnerável neste indicador $(0,159)$, sendo que Fortaleza $(0,272)$ apresenta somente a quarta melhor posição na RMF. As situações mais críticas são encontradas em Aquiraz $(0,931)$, Pindoretama $(0,771)$ e Cascavel $(0,750)$, que apresentam os menores índices de revestimentos entre todas as regiões metropolitanas.

O IVH identifica, para o conjunto da RMF, vulnerabilidade intermediária, devido ao peso de Fortaleza (0,231); com exceção da capital estadual, todos os demais municípios apresentaram maior vulnerabilidade que a média metropolitana.

Para a RMS, apenas Sobral $(0,125)$ e Massapê $(0,188)$ apresentam baixa vulnerabilidade, não se identificando alguma tendência dos municípios limítrofes de Sobral a apresentarem menor vulnerabilidade. São altamente vulneráveis os municípios de Meruoca $(0,507)$ e Pires Ferreira (0,517), apresentando os demais 14 municípios níveis intermediários de vulnerabilidade. A comparação com a RMS evidencia que apenas três municípios registram vulnerabilidade menor que a média metropolitana.

Relativo ao índice sobre as condições de saneamento, somente Sobral $(0,160)$ apresenta baixa vulnerabilidade. Em termos percentuais, 33,3\% dos municípios demonstram elevada vulnerabilidade (em ordem decrescente: Moraújo, Pires Ferreira, Graça, Cariré, Reriutaba e Senador Sá). Destaca-se que dos 10 municípios que estão na faixa intermediária de vulnerabilidade, oito deles $(80 \%)$ apresentam valor acima de 0,4 de vulnerabilidade. Considerando o indicador densidade de dormitório, de forma semelhante ao identificado para Fortaleza, este foi o indicador com maior vulnerabilidade na composição do IVH. Observa-se que os municípios 
mais próximos a Sobral se apresentam como destaques com maiores densidade de pessoas por dormitórios (Forquilha (0,863), Alcântaras $(0,832)$, Coreaú $(0,792)$, Meruoca $(0,771)$, Moraújo $(0,767))$, sendo que Sobral ocupa apenas a oitava posição entre os municípios com maior média de habitantes por dormitório. Para o índice de tipo de revestimento, a RMS apresenta nível intermediário de vulnerabilidade, sendo este o índice com maior percentual de municípios na categoria de baixa vulnerabilidade (50\%). Sobral ocupa somente a $12^{\mathrm{a}}$ melhor posição neste índice.

De modo semelhante ao observado para a RMF, o IVH identifica, para o conjunto da Região Metropolitana de Sobral, vulnerabilidade intermediária $(0,360)$, com sete municípios apresentando vulnerabilidade inferior à média metropolitana (Sobral (0,306), Santana do Acaraú (0,339), Reriutaba (0,352), Pacujá (0,249), Mucambo $(0,346)$, Groaíras $(0,334)$ e Freicheirinha $(0,356))$. Como a maioria destes municípios estão mais afastados de Sobral, percebe-se que não existe para a RMS tendência de aglomeração ou homogeneidade dos municípios próximos ao núcleo dinâmico da RMS.

Na Região Metropolitana do Cariri, para o índice condição de ocupação, percebemse que os valores mais baixos são obtidos pelos municípios que formam a conurbação Crajubar (Crato, Juazeiro do Norte e Barbalha), únicos a apresentarem valores inferiores à média metropolitana, sendo classificados como baixa vulnerabilidade. Nas condições de saneamento dos domicílios, Crato $(0,336)$, Juazeiro do Norte $(0,330)$ e Nova Olinda (0,326) apresentam as menores vulnerabilidades e nenhum município pode ser incluído na classe de baixa vulnerabilidade. Situação mais crítica é encontrada em Caririaçu $(0,517)$, Farias Brito $(0,524)$ e Missão Velha $(0,530)$ com vulnerabilidade alta no respectivo indicador.

Como observado nas demais regiões, o indicador de densidade de dormitórios tem elevada vulnerabilidade para todos os municípios com menor valor para o município do Crato $(0,536)$ e maiores vulnerabilidades relativa à realidade metropolitana para Santana do Cariri $(0,793)$, Caririaçu $(0,737)$ e Jardim $(0,699)$.

Considerando o IVH, a RM Cariri tem vulnerabilidade intermediária (0,334), com o Crajubar apresentando os menores valores. Apesar de Santana do Cariri e Caririaçu terem situação mais crítica relativa ao contexto metropolitano, nenhum destes registra elevada vulnerabilidade.

Na comparação entre as regiões, a RMF apresenta menor valor para o IVH, seguida da RM Cariri. A RMS aponta maiores vulnerabilidade nos indicadores de densidade de dormitórios e de ocupação, enquanto a RMF tem menores vulnerabilidades em condições de ocupação e saneamento, possivelmente este último indicador seja influenciado pelo peso de Fortaleza na composição da RMF, que como capital do estado possui possivelmente maior oferta de serviços de água e esgoto. A RM Cariri se destaca apresentando os valores mais elevados no indicador de condições de saneamento (maior média entre as regiões metropolitanas) e o menor valor para o índice de tipo de revestimento dos domicílios. 


\section{Índice de Vulnerabilidade de Infraestrutura Urbana}

Enquanto o IVH objetiva aferir a vulnerabilidade dos domicílios, o Índice de Vulnerabilidade de Infraestrutura Urbana - IVIU investiga a disponibilidade de serviços públicos de saneamento e abastecimento de água, coleta de lixo, manejo de águas fluviais. Deste modo, enquanto as vulnerabilidades identificadas no IVH atingem domicílios mais pobres, as vulnerabilidades do IVIU podem afetar a população de um modo geral, pois um condomínio de luxo pode não dispor de infraestrutura pública de saneamento e ser obrigado a construir fossas sépticas, com impactos no lençol freático. Os valores estimados para as diferentes regiões também foram ponderados, no caso da taxa de cobertura urbana de esgoto, pela população, para os demais indicadores pelo domicílio.

O indicador de cobertura urbana de esgoto (ICUE) evidencia elevada vulnerabilidade para todas as regiões metropolitanas do estado. $\mathrm{Na} \mathrm{RMF}$, apenas Paraipaba foi classificada como de baixa vulnerabilidade e somente dois municípios foram classificados como vulnerabilidade média ou intermediária, Fortaleza $(0,421)$ e Pacatuba $(0,487)$, ainda assim próximos ao limite superior da referida classe do indicador. Deste modo, 16 dos 19 municípios (84,2\%) são altamente vulneráveis.

\section{Tabela 2 - Índice de Vulnerabilidade de Infraestrutura Urbana e indicadores por Região Metropolitana do Ceará, 2019}

\begin{tabular}{lcccccc}
\hline Municípios & ICUE & IAA & ICL & ICE & IMAP & IVIU \\
\hline Média RMF & 0,501 & 0,123 & 0,055 & 0,406 & 0,611 & 0,339 \\
Fortaleza & 0,421 & 0,041 & 0,000 & 0,384 & 0,600 & 0,289 \\
Média RMS & 0,619 & 0,205 & 0,295 & 0,318 & 0,473 & 0,382 \\
Sobral & 0,314 & 0,031 & 0,087 & 0,288 & 0,600 & 0,264 \\
Média RM Cariri & 0,708 & 0,162 & 0,186 & 0,497 & 0,809 & 0,472 \\
Barbalha & 0,490 & 0,204 & 0,261 & 0,471 & 1,000 & 0,485 \\
Crato & 0,672 & 0,131 & 0,128 & 0,514 & 1,000 & 0,489 \\
Juazeiro do Norte & 0,636 & 0,049 & 0,038 & 0,528 & 0,800 & 0,410 \\
\hline
\end{tabular}

Fonte: Elaborada pelos autores.

Considerando o indicador de abastecimento de água (IAA), a média metropolitana apresenta-se de baixa vulnerabilidade $(0,123)$, porém, há que se pontuar a forte heterogeneidade entre os municípios. Deste modo, apenas quatro municípios apresentam índices menores que a média metropolitana (Maracanaú $(0,0)$, Pacatuba $(0,016)$, Fortaleza $(0,041)$ e Itaitinga $(0,067))$. Além destes, outros dois estão incluídos na faixa de baixa vulnerabilidade (Caucaia $(0,171)$ e Maranguape $(0,157)$. $\mathrm{Na}$ classe de vulnerabilidade intermediária, encontram-se cinco municípios. A situação é crítica para sete municípios que apresentam elevada vulnerabilidade 
(Aquiraz (0,861), Cascavel (0,574), Chorozinho (0,506), Paracurú (0,614), Pindoretama (0,665), São Gonçalo do Amarante (0,520) e Trairi $(0,852))$.

$\mathrm{O}$ indicador de coleta de lixo domiciliar (ICL) apresentou o menor valor entre os indicadores de IVIU, onde Fortaleza apresentou a melhor taxa de cobertura do estado (sem vulnerabilidade); ao todo, nove municípios (47,4\% do total da região) possuem baixa vulnerabilidade. Em nove municípios se constatou vulnerabilidade intermediária. A situação mais vulnerável está no município de Trairi $(0,702)$, com o menor percentual de domicílios com coleta de lixo. O indicador condições de entorno (ICE) para a RMF indica vulnerabilidade intermediária $(0,406)$, onde nenhum município, mesmo a capital estadual, revelou baixa vulnerabilidade, sendo que oito municípios apresentaram vulnerabilidade inferior à média da RM (Fortaleza, Horizonte, Pacajus, Paracuru, Paraipaba, São Gonçalo do Amarante, São Luís do Curú e Trairi). Ademais, quatro municípios possuem elevada vulnerabilidade (Caucaia, Chorozinho, Guaiuba e Itaitinga).

No indicador manejo de águas pluviais (IMAP), percebem-se as maiores vulnerabilidades. Este indicador revela os investimentos no manejo adequado em situação de chuva ou cheias de córregos e rios, com infraestrutura destinada a conter o fluxo de água ou reduzir seu poder destrutivo. A RMF apresenta elevada vulnerabilidade neste indicador $(0,611)$. Somente seis municípios aparecem com vulnerabilidade baixa ou intermediária (Paraipaba, Pacatuba, Cascavel, Trairi, Pindoretama e Pacajus). O restante dos municípios registra elevada vulnerabilidade, sendo que dois deles não apresentaram qualquer infraestrutura prevista, sujeitando sua população à máxima vulnerabilidade (Aquiraz e Maracanaú). O Índice de Vulnerabilidade de Infraestrutura Urbana (IVIU) evidencia, para a RMF, vulnerabilidade média (0,339), já que nenhum dos municípios se classificou com baixa vulnerabilidade. Apresentaram menor vulnerabilidade que a região metropolitana os municípios de Pacatuba, Fortaleza, Paraipaba. Por outro lado, sete municípios (36,8\%) apresentaram vulnerabilidade elevada (Trairi, São Luís do Curu, São Gonçalo do Amarante, Pindoretama, Euzébio, Chorozinho e Aquiraz).

A RMS mostra-se crítica no indicador de cobertura urbana de esgoto (ICUE). Apenas três municípios, Sobral, Alcântaras e Forquilha têm média vulnerabilidade, com os demais sendo de alta vulnerabilidade. Deste grupo, 10 municípios $(66,7 \%)$ possuem máxima vulnerabilidade por não disporem de cobertura urbana de esgoto. Relativo ao abastecimento de água (IAA), observa-se que Sobral registra a menor vulnerabilidade $(0,03)$, destoando significativamente da realidade metropolitana, influenciando para baixo a média regional. Outros três municípios têm baixa vulnerabilidade (Forquilha (0,066), Groaíras (0,130), Varjota $(0,148)$ ). Semelhante ao observado em outros indicadores da RMS, não se percebe uma tendência de redução da vulnerabilidade em torno do núcleo da região metropolitana. Apenas o município de Meruoca apresentou elevada vulnerabilidade no acesso de sua população à água. Os 13 municípios restantes tiveram vulnerabilidade intermediária. 
Na coleta de lixo (ICL), novamente Sobral destoa da realidade metropolitana, com o menor valor para o indicador $(0,087)$, representando o único município em baixa vulnerabilidade. A situação mais crítica é encontrada em Senador Sá, Santana do Acaraú, Pires Ferreira (maior valor 0,765), Graça, Coreaú e Alcântaras, todos com elevada vulnerabilidade. No indicador condições do entorno (ICE), exceção de Mucambo, com elevada vulnerabilidade $(0,529)$, os demais municípios estão na faixa de vulnerabilidade intermediária.

Para os indicadores de manejo de águas fluviais (IMAP), percebe-se que a realidade da RMS diverge consideravelmente das demais regiões metropolitanas do estado, com o maior percentual de municípios na categoria de baixa vulnerabilidade $(50 \%$ possuem valor de 0,2 para o indicador), correspondendo a nove municípios (Alcântaras, Coreaú, Graça, Meruoca, Moraújo e Mucambo, Pires Ferreira, Senador Sá e Varjota). É relevante pontuar que estes municípios também se caracterizam por não possuírem área de risco sujeito a inundação ou deslizamento, e em virtude disso, a vulnerabilidade será menor. Por apresentarem áreas sujeitas a risco por ação de águas fluviais e não disporem de drenagem especial, possuem alta vulnerabilidade: Sobral (0,600), Santana do Acaraú (0,800), Groaíras (0,800), Forquilha (0,600).

Por fim, a análise do Índice de Vulnerabilidade de Infraestrutura Urbana para a RMS indica que a região vulnerabilidade intermediária $(0,382)$, com menor valor para Sobral (0,264). Além de Sobral, somente outros três municípios têm menor vulnerabilidade do que a média metropolitana, Varjota $(0,373)$, Massapê $(0,379)$ e Forquilha (0,324). Dos municípios da RM se destacam seis com elevada vulnerabilidade (Santana do Acaraú, Reriutaba, Pires Ferreira, Meruoca, Groaíras e Cariré).

A análise do indicador de cobertura urbana de esgoto (ICUE) para a RM Cariri, evidencia que, com exceção de Barbalha, a qual apresentou indicador com vulnerabilidade intermediária, todos os demais municípios possuem elevada vulnerabilidade. Destaca-se, porém, que o Crajubar mantém, também para este indicador, a menor vulnerabilidade quando comparada aos demais municípios da RM. A situação que emerge dos dados mostra-se extremamente crítica para os outros seis municípios da RM Cariri, onde quatro deles apresentam máxima vulnerabilidade (Santana do Cariri, Nova Olinda, Farias Brito, Caririaçu), enquanto Jardim e Missão Velha possuem vulnerabilidade acima de 0,9. Apesar deste indicador se apresentar elevado para todas as regiões metropolitanas do estado, no caso da RM Cariri, a disparidade representa evidência de grave deficiência de investimentos em saneamento na região. O índice de abastecimento de água apresenta baixa vulnerabilidade; mais uma vez os menores valores são encontrados nos municípios do Crajubar. Todavia, é relevante destacar que apenas Juazeiro do Norte e Crato apresentaram baixa vulnerabilidade (0,049 e 0,131, respectivamente). As maiores vulnerabilidades são registradas em Santana do Cariri $(0,483)$ e Jardim $(0,593)$, este último como o único município da região a apresentar elevada vulnerabilidade. 
No que tange à coleta de lixo domiciliar (ICL), a evidência para a RM Cariri revela baixa vulnerabilidade. Apenas Crato e Juazeiro do Norte são inferiores à média metropolitana, sendo os únicos com baixa vulnerabilidade. Cinco municípios (55,56\%) aprecem com vulnerabilidade intermediária, enquanto Missão Velha e Jardim têm alta vulnerabilidade. Sobre as condições do entorno (ICE), a média da região evidencia vulnerabilidade no limite superior da classe de vulnerabilidade intermediária. O Crajubar apresenta vulnerabilidade acima da média metropolitana, com Crato e Juazeiro do Norte possuindo elevada vulnerabilidade. Outro município que apresentou elevada vulnerabilidade foi Caririaçu $(0,557)$, representando a máxima vulnerabilidade neste indicador. Os demais municípios apresentaram vulnerabilidade intermediária. No manejo de águas fluviais, a região metropolitana tem elevada vulnerabilidade ${ }^{4}$. As menores vulnerabilidades são encontradas em Santana do Cariri, Nova Olinda e Farias Brito, no nível intermediário $(0,400)$, influenciados pelo fato de não possuírem áreas suscetíveis a inundação ou com baixo risco de deslizamento. Crato e Barbalha apresentam máxima vulnerabilidade (IMAP $=1)$ pela presença de elevado risco de danos causados por águas pluviais e reduzida infraestrutura para conter a força das águas ${ }^{5}$. Outros municípios que apresentam elevada vulnerabilidade $(0,8)$ são Missão Velha, Juazeiro do Norte e Jardim.

Relativo ao Índice de Vulnerabilidade de Infraestrutura Urbana (IVIU), a RM Cariri registra vulnerabilidade média $(0,472)$. As menores vulnerabilidades estão em Juazeiro do Norte $(0,410)$, Nova Olinda $(0,480)$, Barbalha $(0,485)$ e Crato $(0,489)$. Os demais municípios demonstraram elevada vulnerabilidade. Porém, uma comparação entre as regiões metropolitanas do Estado do Ceará para a dimensão de infraestrutura urbana permite identificar que todas estão na faixa de vulnerabilidade intermediária, com menores valores para a RMF e maiores para a RM Cariri.

\section{Índice de Vulnerabilidade por Pressão sobre o Ambiente Natural}

$\mathrm{Na}$ análise da RMF, o indicador de emissão de monóxido de carbono por habitante (IMCV) apresenta valores esperados, onde Fortaleza se destaca pela maior fragilidade $(0,658)$, por se constituir na maior cidade do estado. Os demais municípios têm valores significativamente menores, onde 11 destes $(57,9 \%)$ possuem baixa vulnerabilidade e os demais, vulnerabilidade intermediária, com destaque para Horizonte, Eusébio e Pacajus, que depois de Fortaleza têm as maiores

\footnotetext{
4 A vulnerabilidade regional a este indicador está relacionada ao fato da Região do Cariri apresentar clima tropical subúmido (CEARÁ, 2011) com maior pluviosidade e grande número de áreas de encostas ou sujeitas a deslizamentos, sobretudo nas cidades próximas às encostas da Chapada do Araripe.

5 Destaca-se que para estes municípios a presença de enchentes e deslizamentos é comum todos os anos na quadra chuvosa.
} 
vulnerabilidade relacionadas à poluição do ar. No indicador de resíduos industriais (IRI), a maior vulnerabilidade é encontrada em Maracanaú, representando um dos municípios com maior concentração de indústrias da região metropolitana ${ }^{6}$. A capital estadual também apresentou vulnerabilidade elevada $(0,547)$. Os demais municípios $(89,5 \%)$ revelaram vulnerabilidade baixa.

Considerando focos de queimada (IFQ), os dados mostram a baixa vulnerabilidade da RMF com apenas dois municípios (Cascavel e Guaiúba) de vulnerabilidade intermediária. É importante ressaltar que este indicador deva se mostrar mais expressivo em municípios rurais, explicando a baixa vulnerabilidade nos mais industrializados e com menor área rural. No percentual de área destinada a matas ou florestas (IAP), objetiva-se mensurar a vulnerabilidade advinda da formação de ilhas de calor, evento comum em centros urbanos; com exceção de São Luiz do Curú $(0,355)$ todos os demais municípios apresentaram vulnerabilidades próximas a 1 (valor máximo). Relativo à presença de doenças de notificação compulsória causada por vetor biológico (IDNC), a evidência para a RMF aponta para baixa fragilidade em 18 municípios, apenas Fortaleza tem vulnerabilidade intermediária. Apesar da reduzida vulnerabilidade, destacam-se Fortaleza, Caucaia e São Gonçalo do Amarante com os maiores valores.

\section{Tabela 3 - Índice de Vulnerabilidade por Pressão sobre o Ambiente Natural e indicadores por Região Metropolitana do Ceará, 2019}

\begin{tabular}{lcccccc}
\hline Municípios & IMCV & IRI & IFQ & IAP & IDNC & IVPN \\
\hline Média RMF & 0,501 & 0,427 & 0,153 & 0,893 & 0,178 & 0,430 \\
Fortaleza & 0,658 & 0,547 & 0,056 & 1,000 & 0,243 & 0,501 \\
Média RMS & 0,415 & 0,006 & 0,153 & 0,943 & 0,054 & 0,314 \\
Sobral & 0,589 & 0,012 & 0,102 & 0,951 & 0,066 & 0,344 \\
Média RM Cariri & 0,394 & 0,018 & 0,181 & 0,900 & 0,039 & 0,307 \\
Barbalha & 0,299 & 0,073 & 0,057 & 0,762 & 0,028 & 0,244 \\
Crato & 0,423 & 0,020 & 0,183 & 0,946 & 0,100 & 0,334 \\
Juazeiro do Norte & 0,493 & 0,014 & 0,105 & 0,982 & 0,017 & 0,322 \\
\hline
\end{tabular}

Fonte: Elaborada pelos autores.

Assim, para o Índice de Vulnerabilidade por Pressão sobre o Ambiente Natural IVPN, dois municípios registram vulnerabilidade, São Luiz do Curú e Guaiúba. Isso pode ser devido ao maior afastamento do centro dinâmico da região e por isto apresentam menor atração de atividades industriais. Apenas Fortaleza possui elevada vulnerabilidade por pressão ao ambiente natural, estando os demais

\footnotetext{
6 Como o índice mensurado em kg/hab e este apresenta menor população comparada à capital, explica-se a elevada vulnerabilidade da população deste município.
} 
municípios na faixa intermediária de vulnerabilidade. A RMF registra vulnerabilidade intermediária, porém, com índice próximo à faixa superior da classe.

Para a RMS, o indicador de emissão de monóxido de carbono aponta dois municípios em elevada vulnerabilidade, Sobral e Groaíras, com 0,589 e 0,575, respectivamente. Apenas três municípios possuem baixa vulnerabilidade à poluição do ar (Pires Ferreira, Moraújo e Senador Sá), estando os demais (72,2\%) com vulnerabilidade intermediária. $\mathrm{Na}$ análise do indicador de resíduos industriais, todos os municípios apresentam baixa vulnerabilidade, em virtude da menor concentração industrial em comparação à RMF.

Relativo a focos de queimada, nove municípios têm baixa vulnerabilidade (Sobral, Varjota, Senador Sá, Santana do Acaraú, Meruoca, Moraújo, Massapê, Frecheirinha e Coreaú), situando-se os demais na classe intermediária. Destaque para Mucambo, que apresentou o maior valor para o indicador, estando próximo ao limite superior da classe. Considerando o percentual de áreas de matas ou florestas, a evidência para a RMS é a mesma da RMF, com o agravante de todos os municípios apresentarem elevada vulnerabilidade.

No indicador de presença de doenças de notificação compulsória, todos os municípios registram baixa vulnerabilidade, porém, destaca-se Reriutaba com notificação de casos três vezes maior que a média metropolitana.

O Índice de Vulnerabilidade por Pressão sobre o Ambiente Natural, para a RMS, revela situação intermediária de vulnerabilidade, apesar de apresentar valor significativamente menor que a RMF. Os dados sinalizam homogeneidade entre os municípios, que estão concentrados na mesma faixa da média metropolitana, sendo a maior vulnerabilidade corresponde ao núcleo da região (Sobral $(0,344)$ ).

Para a RM Cariri, o indicador de emissão de monóxido de carbono aponta dois municípios em baixa vulnerabilidade, Farias Brito e Santana do Cariri. Os demais registram vulnerabilidade intermediária, com maiores valores para Juazeiro do Norte e Crato. O indicador de resíduos industriais revela todos os municípios em baixa vulnerabilidade relativa ${ }^{7}$. Em relação ao indicador focos de queimada, a RM Cariri, apesar de revelar situação de baixa vulnerabilidade, possui os maiores valores entre as regiões metropolitanas, isto se deve pela maior presença de municípios rurais, seis dos nove municípios apresentaram baixa vulnerabilidade, enquanto Nova Olinda, Caririaçu e Farias Brito apresentaram níveis intermediários de vulnerabilidade. No que tange ao indicador de percentual de áreas de matas ou florestas, a evidência para a RM Cariri é a mesma das outras regiões metropolitanas, estando todos os municípios em elevada vulnerabilidade.

\footnotetext{
7 Deve-se lembrar que todos os indicadores são construídos levando em consideração a evidência estadual, assim, tais valores são relativamente mais baixos, pois foram comparados com a emissão de resíduos de municípios mais industrializados (Maracanaú, valor máximo do índice em kg/hab.).
} 
Para o indicador de presença de doenças de notificação compulsória, todos os municípios estão em baixa vulnerabilidade, sendo a média da RM Cariri a menor entre as regiões metropolitanas do Ceará. O Índice de Vulnerabilidade por Pressão sobre o Ambiente Natural (IVPN) para a Região Metropolitana do Cariri revela que a região apresenta vulnerabilidade intermediária em todos os municípios, com maiores valores para Crato e Juazeiro do Norte (maiores centros urbanos da região), consolidando a percepção de maior pressão sobre o ambiente natural exercida pelos núcleos das regiões metropolitanas, ressaltando a importância da melhor distribuição dos assentamentos urbanos para a construção da sustentabilidade (SACHS, 2009). É relevante observar que, no conjunto das regiões metropolitanas do Ceará, o Cariri apresenta a menor pressão sobre o ambiente natural, enquanto a RMF apresenta a maior vulnerabilidade neste índice.

\section{Índice Sintético de Vulnerabilidade Ambiental}

$\mathrm{Na}$ Tabela 4, constam os dados do Índice Sintético de Vulnerabilidade Ambiental (ISVA). Para a Região Metropolitana de Fortaleza, todos os municípios apresentam vulnerabilidade intermediária, as maiores disparidades são representadas pelos municípios de Aquiraz $(0,497)$ e Pacatuba $(0,262)$, com vulnerabilidades afastadas mais de dois desvios-padrões da média. Também é possível notar que 84,2\% dos municípios da RMF estão no intervalo de 1 desvio-padrão acima ou abaixo da média, indicando forte homogeneidade entre os municípios em torno da média. Fortaleza, enquanto núcleo dinâmico da RMF, registra valor para o índice ISVA 3,7\% abaixo da média regional. A RMF também apresenta elevada assimetria, com 16 municípios acima da média metropolitana.

Para a Região Metropolitana de Sobral, a classificação em termos de valores do ISVA aponta para vulnerabilidade intermediária, com média muito próxima à observada para a RMF, porém, apresenta menor dispersão, pois o município mais vulnerável é Meruoca, com ISVA igual a 0,424, significativamente a maior vulnerabilidade encontrada na RMS. Em compensação não possui nenhum município com vulnerabilidade inferior a 0,300. Em termos de análise da concentração em torno da média, 72,2\% dos municípios estão no intervalo entre 1 desvio-padrão distante da média, enquanto Sobral, o núcleo da RM, situa-se 13,35\% abaixo da média metropolitana, e 77,8\% dos municípios apresentam vulnerabilidade acima da média regional. 
Tabela 4 - Índice Sintético de Vulnerabilidade Ambiental dos municípios das regiões metropolitanas, 2019

\begin{tabular}{|c|c|c|c|c|c|c|c|c|}
\hline \multicolumn{2}{|l|}{ Municípios } & ISVA & \multicolumn{2}{|l|}{ Municípios } & ISVA & \multicolumn{2}{|l|}{ Municípios } & ISVA \\
\hline \multirow[t]{3}{*}{ Fortaleza } & & \multirow[t]{3}{*}{0,340} & \multirow[t]{3}{*}{ Sobral } & & \multirow[t]{3}{*}{0,305} & \multicolumn{2}{|l|}{ Barbalha } & 0,349 \\
\hline & & & & & & \multicolumn{2}{|l|}{ Crato } & 0,378 \\
\hline & & & & & & \multicolumn{2}{|l|}{ Juazeiro do Norte } & 0,348 \\
\hline \multicolumn{2}{|l|}{ Média RMF } & 0,353 & Média RMS & & 0,352 & Média RM Cariri & & 0,371 \\
\hline \multicolumn{9}{|c|}{ Estatísticas Descritivas } \\
\hline \multicolumn{3}{|c|}{$\begin{array}{c}\text { Intervalos em desvios padrões } \\
\text { da média - RMF }\end{array}$} & \multicolumn{3}{|c|}{$\begin{array}{l}\text { Intervalos em desvios padrões } \\
\text { da média - RMF }\end{array}$} & \multicolumn{3}{|c|}{$\begin{array}{c}\text { Intervalos em desvios padrões } \\
\text { da média - RMF }\end{array}$} \\
\hline 1Desv. Pad. & 0,338 & 0,436 & 1Desv. Pad. & 0,349 & 0,416 & 1Desv. Pad. & 0,364 & 0,426 \\
\hline 2Desv. Pad. & 0,289 & 0,486 & 2Desv. Pad. & 0,315 & 0,450 & 2Desv. Pad. & 0,332 & 0,457 \\
\hline 3Desv. Pad. & 0,239 & 0,535 & 3Desv. Pad. & 0,281 & 0,484 & 3Desv. Pad. & 0,301 & 0,488 \\
\hline
\end{tabular}

Fonte: Elaborada pelos autores.

A Região Metropolitana do Cariri apresentou a maior vulnerabilidade entre as regiões metropolitanas do Ceará $(0,371)$, porém, o município mais vulnerável, Farias Brito $(0,424)$, apresenta vulnerabilidade igual à Meruoca na RMS e inferior à Aquiraz $(0,497)$. Percebe-se, no caso da RM Cariri, que o Crajubar possui os menores índices de vulnerabilidade ambiental (Crato 0,378, Juazeiro do Norte 0,348 e Barbalha 0,349). Apenas Crato tem vulnerabilidade fora do intervalo de um desvio-padrão em torno da média. Relativo às assimetrias regionais, apenas Juazeiro do Norte e Barbalha estão abaixo da média metropolitana.

\section{Considerações finais}

Considerando o Índice de Vulnerabilidade Habitacional, há clara tendência, pelos dados apresentados, dos núcleos dinâmicos das regiões metropolitanas apresentarem menores vulnerabilidades, sobretudo nos indicadores de condições de ocupação, condições inadequadas de saneamento e tipo de revestimento dos domicílios, reflexo dos maiores níveis de renda destes municípios. Porém, tal evidência não se reproduz para o indicador densidade de dormitório, onde os núcleos das regiões metropolitanas apresentaram, em sua maioria, maiores vulnerabilidades que outros municípios. Isto provavelmente se relaciona ao forte fator de atração populacional destas regiões, com aumento da densidade de habitantes por área, com reflexo direto neste indicador. Outro elemento que se destaca é que enquanto na RMF e RMS os núcleos das regiões metropolitanas são bem definidos, constituído por apenas um município. O mesmo não se pode afirmar para a RM Cariri, onde o núcleo dinâmico e relativamente homogêneo é composto pela conurbação Crajubar (Crato, Juazeiro do Norte e Barbalha). 
Em relação ao Índice de Vulnerabilidade de Infraestrutura Urbana, as maiores vulnerabilidades em todas as regiões metropolitanas situam-se nos indicadores de cobertura urbana de esgoto e manejo de águas fluviais, evidenciando maior exposição ao risco destas populações tanto às doenças correlacionadas à qualidade do saneamento básico, bem como aos danos materiais característicos da quadra chuvosa. Neste índice parcial, as menores vulnerabilidades são encontradas próximas às regiões metropolitanas para as variáveis de coleta de lixo e abastecimento de água, serviços estes relacionados, de forma mais explícita, às políticas de atração de investimentos industriais.

No tocante ao Índice de Vulnerabilidade por Pressão sobre o Ambiente Natural, os custos ambientais do desenvolvimento mostram-se mais evidentes onde os núcleos mais dinâmicos das áreas metropolitanas apresentam maior vulnerabilidade, por meio da emissão de monóxido de carbono por habitante (em virtude da maior concentração de atividades industriais e de tráfico de veículos) e menor percentual de áreas destinadas para matas ou florestas. Isso evidencia maior preocupação dos núcleos das regiões metropolitanas com o desenvolvimento das atividades econômicas, mas com ações relativamente incipientes de preservação de cobertura vegetal nas áreas urbanas, o que certamente contribuiria para a mitigação de problemas relacionados ao clima urbano, sobretudo a formação de ilhas de calor.

De modo geral, a análise do Índice de Vulnerabilidade Ambiental aponta níveis de vulnerabilidade semelhantes entre as regiões metropolitanas, todavia, com dispersões distintas. Enquanto para a RMF e RM Cariri apresentam maior homogeneidade entre os municípios, a RMS apresenta menor percentual de municípios abaixo da média regional e maior dispersão dos valores, o que leva ao questionamento sobre a existência de efeitos de espraiamento desta região metropolitana.

\section{Referências}

ADGER, W. N. Vulnerability. Global Environmental Change, v. 16, n. 3, p. 268281, 2006. Disponível

em: https://www.projectenportfolio.nl/images/4/41/Adger_2006.pdf. Acesso em: 21 maio 2020.

ALMEIDA, Lutiane Queiroz de. Vulnerabilidade socioambiental de rios urbanos: bacia hidrográfica do rio Maranguapinho/Região Metropolitana de Fortaleza, Ceará. Rio Claro. 2010. 278f. Tese (Doutorado em Geografia) - Instituto de Geociências e Ciência Exatas/Unesp, 2010. 
ARAÚJO, M. C. C.; CÂNDIDO, G. A. Qualidade de vida e sustentabilidade urbana. Revista HOLOS, Ano 30, Vol. 01, p. 3-19, 2014.

BARCELLOS, F. C.; OLIVEIRA, S. M. M. C. Nova fonte de dados sobre risco ambiental e vulnerabilidade social. In: ENCONTRO DA ASSOCIAÇÃO NACIONAL DE PESQUISA E PÓS-GRADUAÇÃO EM AMBIENTE E SOCIEDADE, 4, 2008, Brasília, DF. Anais [...]. Brasília, DF: ANPPAS, 2008.

BRAGA, Tania Moreira et al. Índices de sustentabilidade municipal: o desafio de mensurar. Belo Horizonte: UFMG/Cedeplar, 2003. 22p. (Texto para discussão n. 225).

BRASIL. Ministério do Meio Ambiente. $\mathbf{1}^{\mathbf{o}}$ Inventário nacional de emissões atmosféricas por veículos automotores rodoviários: relatório final. Brasília/DF: MMA, 2011. Disponível em: https://www.mma.gov.br/estruturas/163/_publicacao/163_publicacao 27072011055200.pdf. Acesso em: 22 maio 2020.

BRASIL. Constituição da República Federativa do Brasil de 1988. Diário Oficial [da] República Federativa do Brasil. Brasília/DF, ano CXXVI, n. 191-A, p. 1, 5 out. 1988. Disponível em: http://www.planalto.gov.br/ccivil_03/constituicao/ constituicao.htm. Acesso em: 15 maio 2020.

BRASIL. Lei complementar n. 14, de 8 de junho de 1973. Estabelece as regiões metropolitanas de São Paulo, Belo Horizonte, Porto Alegre, Recife, Salvador, Curitiba, Belém e Fortaleza. Diário Oficial da União. p. 5585, 11 jun. 1973. Disponível em: https://www2.camara.leg.br/legin/fed/leicom/19701979/leicomplementar-14-8-junho-1973-367020-norma-pl.html. Acesso em: 22 maio 2020.

CEARÁ. Lei complementar n. 168, 27 de dezembro de 2016. Dispõe sobre a criação da Região Metropolitana de Sobral, cria o Conselho de Desenvolvimento e Integração da Região Metropolitana de Sobral. Diário Oficial do Estado. Fortaleza, ano VIII, n. 245, p. 4, 27 dez. 2016. Disponível em: http://www.mpce.mp.br/wp-content/uploads/2017/06/LC-168-2016Regi\%C3\%A3o-Metropolitana-de-Sobral.pdf. Acesso em: 22 maio 2020. 
CEARÁ. Lei Complementar n. 78, 26 de junho de 2009. Dispõe sobre a criação da Região Metropolitana do Cariri, cria o Conselho de Desenvolvimento e Integração e o Fundo de Desenvolvimento e Integração da Região Metropolitana do Cariri FDMC, altera a composição de microrregiões do estado do Ceará e dá outras providências. Diário Oficial do Estado. Fortaleza, ano I, n. 121, p. 1, 3 jul. 2009. Disponível em: http://imagens.seplag. ce.gov.br/PDF/20090703/do20090703p01.pdf. Acesso em: 22 maio 2020.

CEARÁ. Inventário Estadual de resíduos sólidos industriais: Ceará. Fortaleza: Superintendência Estadual do Meio Ambiente, 2004. Disponível em: http://antigo.semace.ce.gov.br/programas/residuos/Inventario.pdf. Acesso em: 22 maio 2020.

CEPAL. Vulnerabilidad sociodemográfica: viejos y nuevos riesgos para comunidades, hogares y personas. Brasília/DF: Comissão Econômica para a América Latina e o Caribe, 2002. Disponível em: https://repositorio.cepal.org/bitstream/handle/11362/13019/ S022114_es.pdf?sequence $=1$ \&isAllowed=y. Acesso em: 22 maio 2020.

CONFALONIERI, U. E. C. Global environmental change and health in Brazil: review of the present situation and proposal for indicators for monitoring these effects. In: HOGAN, D. J.; TOLMASQUIM, M. T. (Org.) Human dimensions of global environmental change: Brazilian perspectives. Rio de Janeiro: Academia Brasileira de Ciências, 2001. Disponível em: http://www.abc.org.br/wpcontent/uploads/2018/07/Livro-Human-Dimensions-of-Global-EnvironmentalChange-Brazilian-Perspectives.pdf. Acesso em: 21 maio 2020.

DESCHAMPS, M. V. Vulnerabilidade socioambiental na Região Metropolitana de Curitiba. Curitiba. 2004. 192f. Tese (Doutorado em Meio Ambiente) - Universidade Federal do Paraná, 2004. Disponível em: https://acervodigital.ufpr.br/bitstream/handle/1884/

531/Marley\%20V.\%20Deschamps.pdf?sequence=2\&isAllowed=y. Acesso em: 21 maio 2020.

GAMBA, C. Avaliação da vulnerabilidade socioambiental no município de São Paulo. In: Encontro Nacional da ANPPAS, 5, 2010, Florianópolis. Anais [...]. Florianópolis: ANPPAS, 2010. 
GAMBA, C.; RIBEIRO, W. C. Indicador e avaliação da vulnerabilidade socioambiental no município de São Paulo. GEOUSP, São Paulo, n. 31, Especial, 2012.

GARCÍA-TORNEL, F. C. Algunas cuestiones sobre geografía de los riesgos. Revista Electrónica de Geografía y Ciencias Sociales, Barcelona, v. 1, n. 1, p. 113, 1997.

GOMES, L. M. S.; GOMES, A. O. Desenvolvimento e análise de um Índice de Qualidade de Vida Urbana. In: VIII Congresso Brasileiro de Gestão Ambiental, 2017, Campo Grande, MS. Anais [...]. Campo Grande/MS: IBEAS, 2017.

HOGAN, D. J.; MARANDOLA Jr., E. Para uma conceituação interdisciplinar da vulnerabilidade. In: CUNHA, J. M. P. (Org.) Novas metrópoles paulistas: população, vulnerabilidade e segregação. Campinas: NEPO/UNICAMP, 2006.

IBGE. Indicadores de desenvolvimento sustentável: Brasil 2015. Rio de Janeiro: Instituto Brasileiro de Geografia e Estatística, 2015, 351p.

IBGE. Censo Demográfico 2010. Rio de Janeiro: Instituto Brasileiro de Geografia e Estatística, 2011. Disponível em: https://censo2010.ibge.gov.br/resultados.html. Acesso em: 22 maio 2020.

IBGE. Pesquisa Nacional de Saneamento Básico: 2008. Rio de Janeiro: Instituto Brasileiro de Geografia e Estatística, 2010. Disponível em: https://biblioteca.ibge.gov.br/visualizacao/livros/liv45351.pdf. Acesso em: 22 maio 2020.

IBGE. Censo Agropecuário 2006. Rio de Janeiro: Instituto Brasileiro de Geografia e Estatística, 2007. Disponível em: https://sidra.ibge.gov.br/pesquisa/censoagropecuario/censo-agropecuario-2006/segunda-apuracao. Acesso em: 22 maio 2020.

INTERGOVERNMENTAL PANEL ON CLIMATE CHANGE. Climate change 2001: impacts, adaptation, and vulnerability. Geneva: IPCC, 2001. 22p. Disponível em: http://www.ipcc.ch. Acesso em: 22 fev. 2014. 
IPEA. Atlas da vulnerabilidade social nos municípios brasileiros. Brasília: Instituto de Pesquisa Econômica Aplicada, 2015. 77p.

IPECE. Anuário estatístico do Ceará 2011. Fortaleza: Instituto de Pesquisa e Estratégia Econômica do Ceará, 2011. Disponível em: http://www2.ipece.ce.gov.br/publicacoes/anuario/anuario2011/index.htm. Acesso em: 22 maio 2020.

IPECE. Regiões metropolitanas do Ceará. Fortaleza: Instituto de Pesquisa e Estratégia Econômica do Ceará, 2019. Disponível em: http://www2.ipece.ce.gov.br/atlas/capitulo1/11/pdf/Regioes_Metropolitanas_d o_Ceara_2019.pdf. Acesso em: 20 jun. 2020.

KAZTMAN, R. et al. Vulnerabilidad, activos y exclusión social en Argentina y Uruguay. Santiago: OIT, 1999. (Documento de Trabajo, 107).

KAZTMAN, R.; FILGUEIRA, F. As normas como bem público e privado: reflexões nas fronteiras do enfoque "ativos, vulnerabilidade e estrutura de oportunidades" (Aveo). In: CUNHA, J. M. P. (Org.) Novas metrópoles paulistas: população, vulnerabilidade e segregação. Campinas: NEPO/UNICAMP, 2006.

MARTINS, M. F.; CÂNDIDO, G. A. Modelo de avaliação do nível de sustentabilidade urbana: proposta para as cidades brasileiras. Revista Brasileira de Gestão Urbana, 7 (3), p. 397-410, 2015.

MENDONÇA, F. Riscos, vulnerabilidade e abordagem socioambiental urbana: uma reflexão a partir da RMC e de Curitiba. Desenvolvimento e Meio Ambiente, Curitiba, n. 10, p. 139-148, 2004. Disponível em: https://revistas.ufpr.br/made/article/download/3102/2483. Acesso em: 21 maio 2020 .

PEREIRA, F. S; VIEIRA, I. C. G. Expansão urbana da Região Metropolitana de Belém sob a ótica de um sistema de índices de sustentabilidade. Revista Ambiente e Água. v. 11, n. 3, p. 731-744, 2016. 
RODRIGUES, A. S.; ALVES, C. L. B.; PINHEIRO, V. F. Dinâmica recente de acumulação do capital e processo de metropolização: o caso da Região Metropolitana do Cariri-CE. In: PINHEIRO, V. F. et al. (Org.) Para pensar o desenvolvimento da RM Cariri. São Paulo: Blucher, 2017. p. 17-50. Disponível em: https://www.blucher.com.br/livro/detalhes/para-pensar-o-desenvolvimentoda-rm-cariri-1331/administracao-123. Acesso em: 21 maio 2020.

ROLNIK, Raquel. Guerra dos lugares: a colonização da terra e da moradia na era das finanças. São Paulo: Boitempo, 2015.

SACHS, I. Caminhos para o desenvolvimento sustentável. Rio de Janeiro: Garamond, 2009. 96p.

TOMINAGA, L. K.; SANTORO, J.; AMARAL, R. (Org.) Desastres naturais: conhecer para prevenir. São Paulo: Instituto Geológico, 2009. Disponível em: https://smastr16.blob.core.

windows.net/publicacoes/2016/12/DesastresNaturais.pdf. Acesso em: 21 maio 2020.

UNDRR. Global assessment report on disaster risk reduction: revealing risk, redefining development. Geneva: UNISDR, 2011.

UNDRR. Living with risk: a global review of disaster reduction initiatives, vol. 2 - annexes. Geneva: UNISDR, 2004.

VEYRET, Y. Os riscos: o homem como agressor e vítima do meio ambiente. São Paulo: Contexto, 2007.

VIGNOLLI, J. R. Vulnerabilidade sociodemográfica: antigos e novos riscos para a América Latina e o Caribe. In: CUNHA, J. M. P. (Org.) Novas metrópoles paulistas: população, vulnerabilidade e segregação. Campinas: NEPO/UNICAMP, 2006. 
Data de submissão: 17/07/2020

Data de aprovação: 09/04/2021

Revisão: Daniela Matthes (português), Anderson de Miranda Gomes (inglês) e Yanet María Reimondo Barrios (espanhol).

\author{
Christiane Luci Bezerra Alves \\ Departamento de Economia / Universidade Regional do Cariri \\ Rua Padre Arnaldo de Melo, 375 - Sossego \\ 63105-170 Crato/CE, Brasil \\ Orcid: http://orcid.org/0000-0001-5987-6814 \\ E-mail: chrisluci@gmail.com

\section{Anderson da Silva Rodrigues} \\ Departamento de Economia / Universidade Regional do Cariri \\ Rua General Raimundo Teles, 39 - Grangeiro \\ 63109-070 Crato/CE, Brasil \\ Orcid: http:/ /orcid.org/0000-0002-2559-0305 \\ E-mail: anderson_rodrigues750@hotmail.com

\section{Nayara de Sousa Rodrigues \\ Bacharel em Ciências Econômicas \\ Travessa Porteiras, 125 - Vila Alta \\ 63119-040 Crato/CE, Brasil} \\ Orcid: http://orcid.org/0000-0002-1903-1195 \\ E-mail: n-sousa-rodrigues@bol.com.br
}

